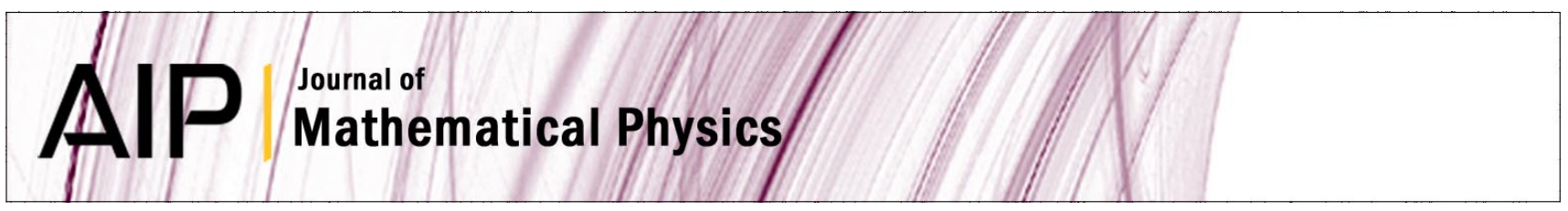

\title{
On construction of recursion operators from Lax representation
}

Metin Gürses, Atalay Karasu, and Vladimir V. Sokolov

Citation: J. Math. Phys. 40, 6473 (1999); doi: 10.1063/1.533102

View online: http://dx.doi.org/10.1063/1.533102

View Table of Contents: http://jmp.aip.org/resource/1/JMAPAQ/v40/i12

Published by the American Institute of Physics.

\section{Additional information on J. Math. Phys.}

Journal Homepage: http://jmp.aip.org/

Journal Information: http://jmp.aip.org/about/about_the_journal

Top downloads: http://jmp.aip.org/features/most_downloaded

Information for Authors: http://jmp.aip.org/authors

\section{ADVERTISEMENT}

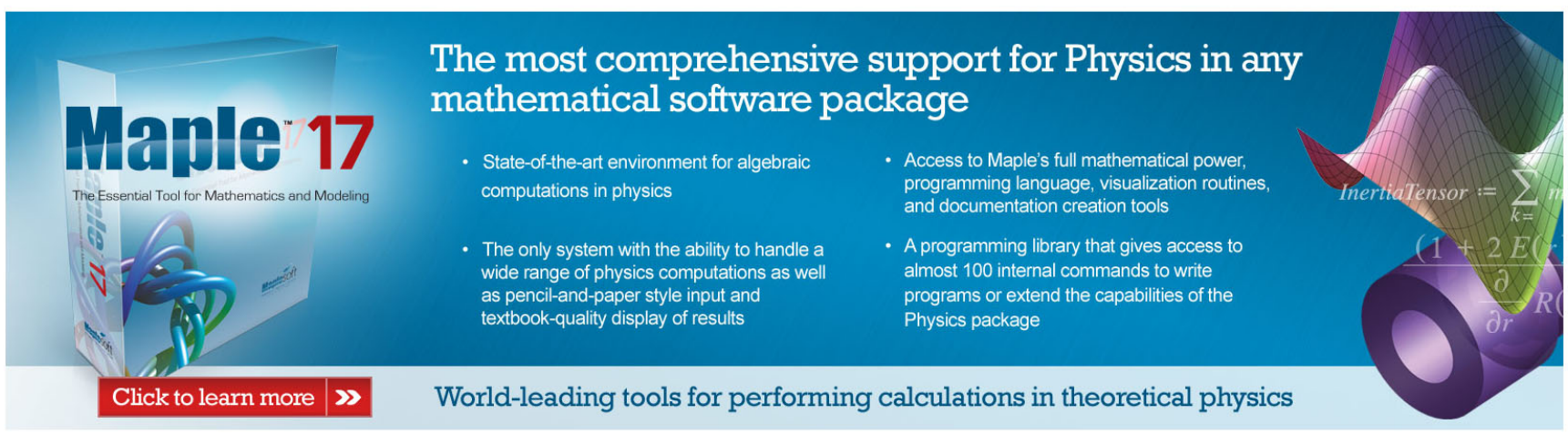




\title{
On construction of recursion operators from Lax representation
}

\author{
Metin Gürses ${ }^{a}$ \\ Department of Mathematics, Faculty of Sciences, Bilkent University, \\ 06533 Ankara-Turkey \\ Atalay Karasu \\ Department of Physics, Faculty of Arts and Sciences, Middle East Technical University, \\ 06531 Ankara-Turkey
}

Vladimir V. Sokolov

Landau Institute, Moscow, 117940 Russia

(Received 28 June 1999; accepted for publication 16 August 1999)

In this work we develop a general procedure for constructing the recursion operators for nonlinear integrable equations admitting Lax representation. Several new examples are given. In particular, we find the recursion operators for some $\mathrm{KdV}$ type systems of integrable equations. (C) 1999 American Institute of Physics.

[S0022-2488(99)03212-0]

\section{INTRODUCTION}

It is well known that most of the integrable nonlinear partial differential equations,

$$
u_{t}=F\left(t, x, u, u_{x}, \ldots, u_{n x}\right)
$$

admit a Lax representation,

$$
L_{t}=[A, L],
$$

so that the inverse scattering method is applicable. The generalized symmetries ${ }^{1}$ of (1) have also Lax representations with the same $L$ operator,

$$
L_{t_{n}}=\left[A_{n}, L\right], \quad n \geqslant 1 .
$$

The recursion operator $\mathcal{R}$, satisfying the equation (see Ref. 2)

$$
\mathcal{R}_{t}+\left[D_{\mathrm{F}}, \mathcal{R}\right]=0,
$$

where $D_{\mathrm{F}}$ is the Frechét derivative of the function $F$, generates symmetries of (1) starting from the simplest ones. In general, $\mathcal{R}$ is a nonlocal operator (a pseudodifferential operator).

The construction of the recursion operator of a given integrable system (1) is not an easy task. Several works are devoted to this subject. Among these works, most of the authors use (4) for the construction of the recursion operator. ${ }^{3-8}$ There are several difficulties in this direct approach. The main problems are the choices of the order of $\mathcal{R}$ and the structure of the nonlocal terms. This is an approach having no relation with the Lax representation (2).

On the other hand, some of the authors used Lax representation for this purpose. Most of these works are related to the squared eigenfunctions of the Lax operator ${ }^{9-13}$ and are based on finding an eigenvalue equation for the squared eigenfunctions of the Lax operator. The operator corresponding to this eigenvalue equation turns out to be the adjoint of the recursion operator.

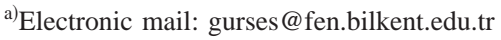


There is an alternative use of the Lax representation to construct recursion operators. This approach is based on the explicit construction of the $A_{n}$ operators (3). It was first used by Symes, ${ }^{14}$ Adler ${ }^{15}$ (see also Dorfman-Fokas, ${ }^{16}$ Fokas-Gel'fand ${ }^{17}$ ) and Antonowicz-Fordy. ${ }^{18,19}$ Although these authors use the Lax representation in different ways, their approach is basically the same. Symes and Adler use the Gel'fand-Dikii ${ }^{20}$ construction of the $A_{n}$ operators. On the other hand, Antonowicz-Fordy determines these operators from integrability condition (3) and by using an ansatz for $A_{n}$. Their basic aim is to determine the Hamiltonian operators $\theta_{1}$ and $\theta_{2}{ }^{21}$ of the equations under consideration. The recursion operator is simply given by $\mathcal{R}=\theta_{2} \theta_{1}^{-1}$. Their approach is based on some explicit formulas for coefficients of the $A_{n}$ operator. This is necessary to find the Hamiltonian operators $\theta_{1}$ and $\theta_{2}$, and it seems that this approach is quite effective to determine the bi-Hamiltonian structure for the simple cases but it becomes more complicated when the $L$-operator has a sophisticated structure.

If one is interested only in the determination of the recursion operator $\mathcal{R}$, we shall show in this work that it is possible to succeed this without any concrete information of the coefficients of $A_{n}$ operators. We use only an ansatz $\widetilde{A}=\mathcal{P} A+R$ that relates $A_{n}$ operators for different $n$. Here $\mathcal{P}$ is some operator that commutes with the $L$ operator and $R$ is the remainder.

We follow this basic idea, partially used by Symes, ${ }^{14}$ Adler. ${ }^{15}$ Shabat and Sokolov, ${ }^{22}$ and establish an extremely simple, effective, and algorithmic method for the construction of recursion operators when the Lax representation (2) is given. ${ }^{23}$

In the next section we consider the case where $L$ is a scalar operator. We first consider the case where $L$ is a differential operator and then the case where it is a pseudodifferential operator. In each case we present our method, discuss the reductions, and give examples for illustrations. In Sec. III we consider Lax operator taking values in a Lie algebra. We give our method both for the general case and also for the reductions. We give one example for each case in the text. Several additional examples are given in the Appendices A, B, and C corresponding to all different cases.

\section{SCALAR LAX REPRESENTATIONS}

First we consider equations with the scalar Lax representations of the form

$$
L_{t}=[A, L],
$$

where $L$ is, in general, a pseudodifferential operator of order $m$ and $A$ is a differential operator whose coefficients are functions of $x$ and $t$.

The different choice of operators $A$ for a given $L$ leads to a hierarchy of nonlinear systems (3). It is well known that one can define operators $A_{n}$ by the following formula: ${ }^{20}$

$$
A_{n}=\left(L^{n / m}\right)_{+},
$$

where $L^{n / m}$ is a pseudodifferential series of the form $L^{n / m}=\sum_{-\infty}^{n} \mathrm{v}_{i} D^{i}$ and $\left(L^{n / m}\right)_{+}=\sum_{i=0}^{n} \mathrm{v}_{i} D^{i}$. Here $v_{i}$ are some concrete functions depending on the coefficients of $L$ and $D$ is the total derivative with respect to $x$.

In Refs. 25 and 26 the relationships between the Kac-Moody algebras and special types of scalar differential and pseudodifferential operators $L$ were established. All corresponding integrable systems are Hamiltonian ones. For most of them a second Hamiltonian structure is not known up to now.

In this section and Appendices A, B, and C we consider the simplest systems from Refs. 25 and 26 as examples and find their recursion operators. In the sequel these examples will be referred to as Drinfeld-Sokolov (DS) systems. It is interesting to note that in all these examples the order of the recursion operator is equal to the Coexter number of the corresponding KacMoody algebra. 


\section{A. Gel'fand-Dikii systems}

In this section we shall consider the case where $L$ is a differential operator,

$$
L=D^{m}+u_{m-2} D^{m-2}+\cdots+u_{0},
$$

where $u_{i}, i=0,1, \ldots, m-2$ are functions of $x, t$. In the framework of Ref. 25, this corresponds to the Kac-Moody algebras of the type $A_{m-1}^{(1)}$.

To show that (3) is equivalent to a system of $(m-1)$ evolution equations with respect to $u_{i}$ one can use the following standard reasoning. Set

$$
L^{n / m}=\left(L^{n / m}\right)_{+}+\left(L^{n / m}\right)_{-},
$$

where $\left(L^{n / m}\right)_{+}$is the differential part of the series $L^{n / m}$ and $\left(L^{n / m}\right)_{-}$is a series of order $\leqslant-1$. Since $\left[L, L^{n / m}\right]=0$ we have

$$
\left[\left(L^{n / m}\right)_{+}, L\right]=\left[L,\left(L^{n / m}\right)_{-}\right]
$$

The left-hand side of (9) is a differential operator, but the right side is a series of order $\leqslant n-2$. Thus, both sides of (3) are differential operators of order $\leqslant n-2$ and it is equivalent to a system of evolution equations for the dependent variables $u_{i}, i=0,1, \ldots, m-2$. This system can be obtained by comparing the coefficients of $D^{i}$, where $0, \ldots, m-2$ in (3).

Since $L^{(n+m) / m}=L L^{n / m}$, then we have

$$
A_{m+n}=\left(L L^{n / m}\right)_{+}=L\left(L^{n / m}\right)_{+}+\left(L\left(L^{n / m}\right)_{-}\right)_{+}
$$

which leads directly to

$$
L_{t_{n+m}}=\left[A_{n+m}, L\right]=L L_{t_{n}}+\left[\left(L\left(L^{n / m}\right)_{-}\right)_{+}, L\right]
$$

The above equation (11) has been given also by Symes $^{14}$ (see also Adler's paper ${ }^{15}$ ). In his work Symes expressed the coefficients of the both parts of (11), in a rather complicated way, in terms of some finite set of coefficients of the resolvent for an $L$ operator. That allows him to express $L_{t_{n+m}}$ in terms of $L_{t_{n}}$. This relation gives directly the recursion operator. He gave explicit formulas for the cases $m=2$ and $m=3$.

In this section we shall show that in order to construct the recursion operator it suffices to know only that

$$
L_{t_{n+m}}=L L_{t_{n}}+\left[R_{n}, L\right]
$$

Obviously, it follows from the following.

Proposition 1: For any n,

$$
A_{n+m}=L A_{n}+R_{n}
$$

where $R_{n}$ is a differential operator of order $\leqslant m-1$.

Proof: The relation (13) coincides with (10) if we put

$$
R_{n}=\left(L\left(L^{n / m}\right)_{-}\right)_{+} \cdot
$$

Since $\left(L^{n / m}\right)_{-}$is a series of order $\leqslant-1$, then $\operatorname{ord}\left(R_{n}\right) \leqslant m-1$.

Remark 1: It follows from the formula

$$
A_{n+m}=\left(L^{n / m} L\right)_{+}=\left(L^{n / m}\right)_{+} L+\left(\left(L^{n / m}\right)_{-} L\right)_{+},
$$

that 


$$
A_{n+m}=A_{n} L+\bar{R}_{n},
$$

and

$$
L_{t_{n+m}}=L_{t_{n}} L+\left[L, \bar{R}_{n}\right]
$$

where $\bar{R}_{n}$ is a differential operator of order $\leqslant m-1$.

To find the recursion operator we can simply equate the coefficients of different powers of $D$ in (12). It is easy to see that in this comparison of the coefficients of $D^{i}, i=2 m-2, \ldots, m-1$ we determine $R_{n}$ in terms of the coefficients of operators $L$ and $L_{t_{n}}$. It is important that the resulting formulas turn out to be linear in the coefficients of $L_{t_{n}}$. The remaining coefficients of $D^{i}, i=m$ $-2, \ldots, 0$ in (12) give us the relation

$$
\left(\begin{array}{c}
u_{0} \\
\cdot \\
\cdot \\
\cdot \\
u_{m-2}
\end{array}\right)_{t_{n+m}}=\mathcal{R}\left(\begin{array}{c}
u_{0} \\
\cdot \\
\cdot \\
\cdot \\
u_{m-2}
\end{array}\right)_{t_{n}},
$$

where $\mathcal{R}$ is a recursion operator. Instead of (12) one can use (17). The corresponding recursion operators coincide.

Example 1. KdV equation: The KdV equation,

$$
u_{t}=\frac{1}{4}\left(u_{3 x}+6 u u_{x}\right) \text {, }
$$

has a Lax representation with

$$
L=D^{2}+u, \quad A=\left(L^{3 / 2}\right)_{+} .
$$

Since in this case $L_{t_{n+2}}=u_{t_{n+2}} \equiv u_{n+2}$ and $L_{t_{n}}=u_{t_{n}} \equiv u_{n}$, the main relation (12) takes the form

$$
u_{n+2}=\left(D^{2}+u\right) \cdot u_{n}+\left[R_{n}, L\right] \text {, }
$$

with $R_{n}=a_{n} D+b_{n}$.

Now if we equate successively to zero the coefficients of $D^{2}, D$, and $D^{0}$ in the above equation, we obtain

$$
a_{n}=\frac{1}{2} D^{-1}\left(u_{n}\right), \quad b_{n}=\frac{3}{4} u_{n},
$$

and

$$
u_{n+2}=\left(\frac{1}{4} D^{2}+u+\frac{1}{2} u_{x} D^{-1}\right) u_{n},
$$

that gives the standard recursion operator for the $\mathrm{KdV}$ equation,

$$
\mathcal{R}=\frac{1}{4} D^{2}+u+\frac{1}{2} u_{x} D^{-1} .
$$

In the same way one can find a recursion operator for the Boussinesq equation (see Appendix A).

\section{B. Symmetric and skew-symmetric reductions of a differential Lax operator}

The standard reductions of the Gel'fand-Dikii systems are given by the conditions $L^{*}=L$ or $L^{*}=-L$. Here ${ }^{*}$ denotes the adjoint operation defined as follows. Let $L$ be a differential operator, 
$L=\sum a_{i} D^{i}$. Its adjoint $L^{*}$ is given by $L^{*}=\Sigma(-D)^{i} \cdot a_{i}$. It is easy to see that if $L^{*}=L$ then $m$ $=\operatorname{ord}(L)$ must be an even integer. For the case $L^{*}=-L$, it must be an odd integer.

It is well known that for both reductions all possible $A_{n}$ are defined by (6), where $n$ takes odd integer values. This condition provides that $\left(A_{n}\right)^{*}=-A_{n}$ that is necessary for (3) to be compatible.

If $L^{*}=L$, the formula $A_{n+m}=\left(L L^{n / m}\right)_{+}=\left(L^{(n+m) / m}\right)_{+}$gives a correct $A_{n}$ operator since $n$ $+m$ is an odd integer. Thus, in this case Proposition 1 remains valid and the recursion operator can be found from (12) or (17).

On the other hand, if $L^{*}=-L$ then both integers $m$ and $n$ are odd and hence their sum $m$ $+n$ is an even integer. This means that $\left(L^{(n+m) / m}\right)_{+}$cannot be taken as an $A_{n}$ operator. In this (skew adjoint) case we must take

$$
A_{n+2 m}=\left(L^{(n+2 m) / m}\right)_{+}=\left(L^{2} L^{n / m}\right)_{+},
$$

to find the recursion operator. Following the proof of Proposition 1 we obtain Proposition 2.

Proposition 2: If $L^{*}=-L$ then

$$
A_{n+2 m}=L^{2} A_{n}+R_{n},
$$

where $\operatorname{ord}\left(R_{n}\right)<2 \operatorname{ord}(L)$. It follows from (23) that

$$
L_{t_{n+2 m}}=L^{2} L_{t_{n}}+\left[R_{n}, L\right]
$$

Remark 2: Instead of (23) we can use the ansatz

$$
A_{n+2 m}=L A_{n} L+\widetilde{R}_{n},
$$

or

$$
A_{n+2 m}=A_{n} L^{2}+\widetilde{R}_{n} .
$$

The recursion operators obtained by the utility of (23), (25), and (26) all coincide.

In the works, ${ }^{25,26}$ more general reductions $L^{\dagger}= \pm L$ were also considered. Here $L^{\dagger}$ $=K L^{*} K^{-1}$, where $K$ is a given differential operator, such that $L K^{-1}$ is a differential operator. In this general reductions, as well, possible $A_{n}$ operators are given by (6), with $n$ being an odd integer. Propositions 1 and 2 are valid for this general symmetric and skew-symmetric cases and hence one can use Eqs. (12), (24) accordingly to obtain the recursion operators.

Example 2. Kupershmidt equation: This equation,

$$
u_{t}=u_{5 x}+10 u u_{3 x}+25 u_{x} u_{2 x}+20 u^{2} u_{x},
$$

has the Lax pair

$$
L=D^{3}+2 u D+u_{x}, \quad A=\left(L^{5 / 3}\right)_{+} .
$$

In this case $L^{*}=-L$; therefore we use Eq. (24) with

$$
\widetilde{R}_{n}=a_{n} D^{5}+b_{n} D^{4}+c_{n} D^{3}+d_{n} D^{2}+e_{n} D+f_{n} .
$$

By equating the coefficients of powers of $D$ in (24), we obtain

$$
\begin{gathered}
a_{n}=\frac{2}{3} D^{-1}\left(u_{n}\right), \quad b_{n}=\frac{11}{3} u_{n}, \quad c_{n}=\frac{1}{9}\left(20 u D^{-1}\left(u_{n}\right)+73 u_{n, x}\right), \\
d_{n}=\frac{1}{3}\left(10 u_{x} D^{-1}\left(u_{n}\right)+22 u u_{n}+27 u_{n, 2 x}\right),
\end{gathered}
$$




$$
\begin{gathered}
e_{n}=\frac{1}{27}\left(70 u_{2 x} D^{-1}\left(u_{n}\right)-2 D^{-1}\left(u_{2 x} u_{n}\right)+40 u^{2} D^{-1}\left(u_{n}\right)-8 D^{-1}\left(u^{2} u_{n}\right)\right. \\
\left.+134 u_{n, 3 x}+212 u u_{n, x}+184 u_{x} u_{n}\right), \\
f_{n, x}=\frac{1}{27}\left(20 u_{4 x} D^{-1}\left(u_{n}\right)+74 u_{3 x} u_{n}+126 u_{2 x} u_{n, x}+40 u u_{2 x} D^{-1}\left(u_{n}\right)+40 u_{x}^{2} D^{-1}\left(u_{n}\right)\right. \\
\left.+136 u_{x} u_{n, 2 x}+27 u u_{x} u_{n}+28 u_{n, 5 x}+64 u u_{n, 3 x}+16 u^{2} u_{n, x}\right),
\end{gathered}
$$

and the recursion operator for the Kupershmidt equation:

$$
\begin{aligned}
\mathcal{R}= & D^{6}+12 u D^{4}+36 u_{x} D^{3}+\left(49 u_{2 x}+36 u^{2}\right) D^{2}+5\left(7 u_{3 x}+24 u u_{x}\right) D+13 u_{4 x}+82 u u_{2 x}+69 u_{x}^{2} \\
& +32 u^{3}+2 u_{x} D^{-1}\left(u_{2 x}+4 u^{2}\right)+2\left(u_{5 x}+10 u u_{3 x}+25 u_{x} u_{2 x}+20 u^{2} u_{x}\right) D^{-1} .
\end{aligned}
$$

\section{Pseudodifferential Lax operator}

In this section we generalize our scheme to the case of pseudodifferential Lax operators. The only difference is that in formulas like (13) and (23) the $R_{n}$ operator also becomes a pseudodifferential operator.

It follows from these formulas that the structure of the nonlocal terms in $R_{n}$ is, in general, similar to the nonlocal terms in $L$ since $A_{n+m}$ and $A_{n}$ are differential operators.

For skew-symmetric case, $A_{n}$ may be defined by either (23) or (25), or (26). In the pseudodifferential case they are not equivalent, in the sense that the nonlocal part of $R_{n}$ depends on which ansatz we choose. For illustration, let us consider the case $L=M D^{-1}$, where $M$ is a differential operator. The following lemma shows that if $L^{\dagger}=L$ or $L^{\dagger}=-L$, where

$$
L^{\dagger}=D L^{*} D^{-1},
$$

then the formulas (13) and (25) are much suitable then (16), (23), and (26).

Lemma: Let $L^{\dagger}=\epsilon L$, where $\epsilon= \pm 1$. Then

$$
R_{n}=D^{m-1}+\cdots+a_{0}, \quad \text { for } \epsilon=1,
$$

where $R_{n}$ is defined by (13), and

$$
\widetilde{R}_{n}=D^{2 m-1}+\cdots+a_{-1} D^{-1}, \quad \text { for } \epsilon=-1,
$$

where $\widetilde{R}_{n}$ is defined by $(25)$.

Proof: If $L=M D^{-1}$ then $L^{\dagger}=\epsilon L$ implies $M^{*}=-\epsilon M$. It is easy to show that $\left(L^{1 / m}\right)^{\dagger}$ $=-L^{1 / m}$. Hence $\left(L^{n / m}\right)^{\dagger}=-L^{n / m}$ for an odd integer $n$. Define now a series $K_{n}$ by

$$
L^{n / m}=D K_{n} .
$$

It is easy to prove that $K_{n}^{*}=K_{n}$. Since $K_{n}=\left(K_{n}\right)_{+}+\left(K_{n}\right)_{-}$and $\left(K_{n}\right)^{*}=K_{n}$, we have

$$
\left(K_{n}\right)_{+}^{*}=\left(K_{n}\right)_{+}, \quad\left(K_{n}\right)_{-}^{*}=\left(K_{n}\right)_{-} .
$$

From the last formula it follows that $\operatorname{ord}\left(K_{n}\right)_{-} \leqslant-2$, which leads to an important result,

$$
A_{n}=\left(L^{n / m}\right)_{+}=D\left(K_{n}\right)_{+} .
$$

This implies that

$$
L A_{n}=M\left(K_{n}\right)_{+}
$$

is a differential operator. Now using (34) in (13) and (25) for the cases $\epsilon=1$ and $\epsilon=-1$, respectively, we find the ansatz for $A_{n}$ given by (32) and (33). 
Example $3(\epsilon=-1)$ : It is known that the KdV equation has, besides the standard Lax representation, the following Lax pair:

$$
L=\left(D^{2}+u\right) D^{-1}, \quad A=\left(L^{3}\right)_{+} .
$$

The $L$ operator satisfies the reduction $L^{\dagger}=-L$. According to the formula (33) we have

$$
\widetilde{R}_{n}=a_{n} D+b_{n}+c_{n} D^{-1} .
$$

It follows from (25) that

$$
a_{n}=D^{-1}\left(u_{n}\right), \quad b_{n}=u_{n}, \quad c_{n}=-u_{n, x}-u D^{-1}\left(u_{n}\right) .
$$

The remaining equation in (25) gives the recursion operator

$$
\mathcal{R}=D^{2}+4 u+2 u_{x} D^{-1} .
$$

Example $4(\epsilon=1)$. DSIII system: The DSIII system ${ }^{25,26}$ is given by

$$
\begin{gathered}
u_{t}=-u_{3 x}+6 u u_{x}+6 \mathrm{v}_{x}, \\
\mathrm{v}_{t}=2 \mathrm{v}_{3 x}-6 u \mathrm{v}_{x}
\end{gathered}
$$

The nonlocal Lax representation for this system is

$$
\begin{gathered}
L=\left(D^{5}-2 u D^{3}-2 D^{3} u-2 D w-2 w D\right) D^{-1}, \\
A=\left(L^{3 / 4}\right)_{+},
\end{gathered}
$$

where $w=v-u_{2 x}$. Since $L^{\dagger}=L$ we can use (32), which gives us

$$
R_{n}=a_{n} D^{3}+b_{n} D^{2}+c_{n} D+d_{n} .
$$

By equating the coefficients of the powers of $D$ in (25), we obtain

$$
\begin{gathered}
a_{n}=D^{-1}\left(u_{n}\right), \quad b_{n}=4 u_{n}, \\
c_{n}=\frac{1}{2}\left(-6 u D^{-1}\left(u_{n}\right)+11 u_{n, x}+2 D^{-1}\left(u u_{n}\right)+2 D^{-1}\left(\mathrm{v}_{n}\right)\right), \\
d_{n, x}=-\frac{1}{2}\left(6 u_{2 x} D^{-1}\left(u_{n}\right)+10 u_{x} u_{n}-5 u_{n, 3 x}+4 u u_{n, x}-6 \mathrm{v}_{n, x}\right) .
\end{gathered}
$$

The recursion operator of the DSIII is found as

$$
\mathcal{R}=\left(\begin{array}{ll}
\mathcal{R}_{0}^{0} & \mathcal{R}_{1}^{0} \\
\mathcal{R}_{0}^{1} & \mathcal{R}_{1}^{1}
\end{array}\right),
$$

with

$$
\begin{gathered}
\mathcal{R}_{0}^{0}=D^{4}-8 u D^{2}-12 u_{x} D-8 u_{2 x}+16 u^{2}+16 \mathrm{v}+\left(-2 u_{3 x}+12 u u_{x}+12 \mathrm{v}_{x}\right) D^{-1}+4 u_{x} D^{-1} u \\
\mathcal{R}_{1}^{0}=-10 D^{2}+8 u+4 u_{x} D^{-1} \\
\mathcal{R}_{0}^{1}=10 \mathrm{v}_{x} D+12 \mathrm{v}_{2 x}+\left(4 \mathrm{v}_{3 x}-12 u \mathrm{v}_{x}\right) D^{-1}+4 \mathrm{v}_{x} D^{-1} u \\
\mathcal{R}_{1}^{1}=-4 D^{4}+16 u D^{2}+8 u_{x} D+16 \mathrm{v}+4 \mathrm{v}_{x} D^{-1}
\end{gathered}
$$

This recursion operator has recently been given in Ref. 6 . 


\section{MATRIX L OPERATOR OF THE FIRST ORDER}

In this section we demonstrate how our approach, given in the previous sections, can be generalized to the case where $L$ is a matrix operator of the form

$$
L=D_{x}+\lambda a+q(x, t) .
$$

\section{A. General case}

Let us consider the Lax operator (42), where $q$ and $a$ belong to a Lie algebra $g$ and $\lambda$ is the spectral parameter. The constant element $a$ is supposed to be such that

$$
g=\operatorname{Ker}\left(\operatorname{ad}_{a}\right) \oplus \operatorname{Im}\left(\operatorname{ad}_{a}\right) .
$$

First, let us recall the procedure ${ }^{25}$ of constructing the $A$ operators for the Lax operator (42).

Proposition 3: There exist unique series,

$$
\begin{gathered}
u=u_{-1} \lambda^{-1}+u_{-2} \lambda^{-2}+\cdots, \quad u_{i} \in \operatorname{Im}\left(\operatorname{ad}_{a}\right), \\
h=h_{0}+h_{-1} \lambda^{-1}+h_{-2} \lambda^{-2}+\cdots, \quad h_{i} \in \operatorname{Ker}\left(\operatorname{ad}_{a}\right),
\end{gathered}
$$

such that

$$
e^{\mathrm{ad}_{u}(L)}=L+[u, L]+\frac{1}{2}[u,[u, L]]+\cdots=D_{x}+a \lambda+h .
$$

Let $b$ be a constant element of $g$ such that $\left[b, \operatorname{Ker}\left(\operatorname{ad}_{a}\right)\right]=\{0\}$. It follows from (45) that $\left[b \lambda^{n}, D_{x}+a \lambda+h\right]=0$. Hence $\left[\Phi_{b, n}, L\right]=0$, where

$$
\Phi_{b, n}=e^{-\mathrm{ad}_{u}\left(b \lambda^{n}\right) .}
$$

Then the corresponding $A$ operator of the form

$$
A_{b, n}=b \lambda^{n}+a_{n-1} \lambda^{n-1}+\cdots+a_{0},
$$

is defined by the formula

$$
A_{b, n}=\left(\Phi_{b, n}\right)_{+},
$$

where

$$
\left(\Sigma_{-\infty}^{n} \alpha_{i} \lambda^{i}\right)_{+}=\Sigma_{0}^{n} \alpha_{i} \lambda^{i}
$$

According to (47),

$$
\Phi_{b, n+1}=\lambda \Phi_{b, n} .
$$

Hence

$$
A_{b, n+1}=\left(\lambda \Phi_{b, n}\right)_{+}=\lambda\left(\Phi_{b, n}\right)_{+}+\left(\lambda\left(\Phi_{b, n}\right)_{-}\right)_{+} .
$$

The last formula shows that

$$
A_{b, n+1}=\lambda A_{b, n}+R_{n}, \quad R_{n} \in g,
$$

where $R_{n}$ does not depend on $\lambda$. Substituting (53) into the Lax equation $L_{t_{n+1}}=\left[A_{b, n+1}, L\right]$, we get

$$
L_{t_{n+1}}=\lambda L_{t_{n}}+\left[R_{n}, L\right] .
$$


Using the ansatz (54), one can easily find the corresponding recursion operator.

Example 5: The system

$$
\begin{gathered}
u_{t}=-\frac{1}{2} u_{x x}+u^{2} \mathbf{v}, \\
\mathbf{v}_{t}=\frac{1}{2} \mathbf{v}_{x x}-\mathrm{v}^{2} u,
\end{gathered}
$$

is equivalent to the nonlinear Schrödinger equation, has a Lax operator

$$
L=D+\left(\begin{array}{cc}
1 & 0 \\
0 & -1
\end{array}\right) \lambda+\left(\begin{array}{cc}
0 & u \\
\vee & 0
\end{array}\right)
$$

The Lie algebra $g$ in this example coincides with $s l(2)$.

Using (54) with

$$
R_{n}=\left(\begin{array}{cc}
a_{n} & b_{n} \\
c_{n} & -a_{n}
\end{array}\right)
$$

we find that

$$
\begin{aligned}
& a_{n}=\frac{1}{2} D^{-1}\left(\mathrm{v} u_{n}+u \mathrm{v}_{n}\right), \\
& b_{n}=\frac{1}{2} u_{n}, \quad c_{n}=-\frac{1}{2} \mathrm{v}_{n},
\end{aligned}
$$

and the recursion operator of the system (55) is given by

$$
\mathcal{R}=\left(\begin{array}{cc}
-\frac{1}{2} D+u D^{-1} \mathrm{v} & u D^{-1} u \\
-\mathrm{v} D^{-1} \mathrm{\vee} & \frac{1}{2} D-\mathrm{v} D^{-1} u
\end{array}\right) .
$$

\section{B. Reductions in matrix case}

In the general case considered in the previous section the $A_{n}$ operators belong to the Lie algebra,

$$
\mathfrak{a}_{+}=\left\{\sum_{i=0}^{\kappa} a_{i} \lambda^{i}, \quad a_{i} \in g, \kappa \in Z_{+}\right\},
$$

that is a subalgebra of the Lie algebra,

$$
\mathfrak{a}=\left\{\Sigma_{-\infty}^{\kappa} a_{i} \lambda^{i}, \quad \alpha_{i} \in g, \quad \kappa \in Z\right\} .
$$

A standard $\sigma$ reduction is defined by any automorphism $\sigma$ of the Lie algebra $g$ of finite order $\kappa$. Because $\sigma^{\kappa}=\mathrm{Id}$, the eigenvalues of $\sigma$ are $\epsilon^{i}, i=0, \ldots, \kappa-1$, where $\epsilon$ is a primitive $\kappa$ root of unity.

Let $g_{i}$ be an eigenspace corresponding to eigenvalue $\epsilon^{i}$. Then the following reduction $a_{j}$ $\in g_{i}$, where $i=j(\bmod \kappa)$ in (58) and (59) is compatible with Eqs. (3). Note that according to this definition $a \in g_{1}$, and the potential $q(x, t)$ in (42) belongs to $g_{0}$ or, the same, satisfies $\sigma(q)=q$.

It is easy to see that, to satisfy such a reduction, we must use the ansatz

$$
A_{b, n+\kappa}=\lambda^{\kappa} A_{b, n}+R_{n}
$$

where

$$
R_{n}=r_{\kappa-1} \lambda^{\kappa-1}+\cdots+r_{0}, \quad r_{i} \in g_{i} .
$$

Further generalizations are associated with modifications of sign " + " in (50), which corresponds to the simplest decomposition of algebra $\mathfrak{a}$ into the direct sum of two subalgebras, 


$$
\mathfrak{a}=\mathfrak{a}_{+} \oplus \mathfrak{a}_{-},
$$

where $\mathfrak{a}_{+}$is given by $(58)$ and

$$
\mathfrak{a}_{-}=\left\{\Sigma_{-\infty}^{-1} a_{i} \lambda^{i}, \quad a_{i} \in g\right\} .
$$

The sign " + ", in (50) is the projection of onto $\mathfrak{a}_{+}$parallel to $\mathfrak{a}_{-}$. If we have a different decomposition (62), then the construction from Proposition 3 is also valid, but we have the following condition:

$$
R_{n} \in \mathfrak{a}_{+} \cap \lambda \mathfrak{a}_{-},
$$

instead of $R_{n} \in g$. If we also have the $\sigma$ reduction, we must use the most general ansatz (60), where

$$
R_{n} \in \mathfrak{a}_{+} \cap \lambda^{\kappa} \mathfrak{a}_{-} .
$$

Example 6: Let us consider the following equation:

$$
u_{t}=\frac{1}{4} u_{x x x}-\frac{3}{8} u_{x x} u+\frac{3}{8} u u_{x x}-\frac{3}{8} u u_{x} u,
$$

where $u$ is a square matrix of arbitrary size, or more generally, $u$ belongs to an arbitrary associative algebra $\mathcal{K}$. This equation has a Lax representation with

$$
L=D+\left(\begin{array}{ll}
0 & \mathbf{1} \\
\mathbf{1} & 0
\end{array}\right) \lambda+\left(\begin{array}{ll}
u & 0 \\
0 & 0
\end{array}\right)
$$

Here 1 is the unity of $\mathcal{K}$. The reduction (67) can be described as follows (see Ref. 27). The Lie algebra $g$ is the algebra of all $2 \times 2$ matrices with entries belonging to $\mathcal{K}$. The automorphism $\sigma$ is defined by

$$
\sigma(X)=T X T^{-1}
$$

where

$$
T=\left(\begin{array}{cc}
\mathbf{1} & 0 \\
0 & -\mathbf{1}
\end{array}\right)
$$

Obviously $\sigma^{2}=\mathrm{Id}$ and eigenvalues of $\sigma$ are 1 and -1 . The corresponding eigenspaces are

$$
g_{0}=\left\{\begin{array}{cc}
* & 0 \\
0 & *
\end{array}\right\}, \quad g_{1}=\left\{\begin{array}{ll}
0 & * \\
* & 0
\end{array}\right\},
$$

and therefore the coefficients $a_{i}$ in (59) have the following structure:

$$
a_{2 j}=\left(\begin{array}{cc}
* & 0 \\
0 & *
\end{array}\right), \quad a_{2 j+1}=\left(\begin{array}{cc}
0 & * \\
* & 0
\end{array}\right) .
$$

The subalgebra $\mathfrak{a}_{+}$is given by (58), where the coefficients have the structure (70) and, additionally,

$$
a_{0}=\left(\begin{array}{cc}
* & 0 \\
0 & 0
\end{array}\right) \text {. }
$$

The subalgebra $\mathfrak{a}_{-}$has the following form: 


$$
\mathfrak{a}_{-}=\Sigma_{-\infty}^{0} a_{i} \lambda^{i}
$$

where $a_{0}$ is of the form

$$
a_{0}=\left(\begin{array}{ll}
\alpha & 0 \\
0 & \alpha
\end{array}\right), \quad \alpha \in \mathcal{K}
$$

The $A$ operator for (66) is given by formula $A=\left(\Phi_{a, 3}\right)_{+}[$see (49)], where

$$
a=\left(\begin{array}{ll}
0 & \mathbf{1} \\
\mathbf{1} & 0
\end{array}\right)
$$

and " + " " means the projection onto $\mathfrak{a}_{+}$parallel to $\mathfrak{a}_{-}$.

According to $(65), R_{n}$ is of the form

$$
R_{n}=\left(\begin{array}{cc}
a_{n} & 0 \\
0 & a_{n}
\end{array}\right) \lambda^{2}+\left(\begin{array}{cc}
0 & b_{n} \\
c_{n} & 0
\end{array}\right) \lambda+\left(\begin{array}{cc}
d_{n} & 0 \\
0 & 0
\end{array}\right) .
$$

It follows from

$$
L_{t_{n+2}}=\lambda^{2} L_{t_{n}}+\left[R_{n}, L\right]
$$

that

$$
\begin{gathered}
u_{n}-a_{n, x}+\left[a_{n}, u\right]+b_{n}-c_{n}=0 \quad c_{n}-b_{n}-a_{n, x}=0, \\
d_{n}-b_{n, x}-u b_{n}=0, \quad d_{n}+c_{n, x}-c_{n} u=0, \\
u_{n+2}=-d_{n, x}+\left[d_{x}, u\right] .
\end{gathered}
$$

Finding $a_{n}, b_{n}, c_{n}$, and $d_{n}$ from this system, we obtain the following recursion operator:

$$
\mathcal{R}=-\left(D+\mathrm{ad}_{u}\right)\left(-D+R_{u}\right)\left(2 D+\mathrm{ad}_{u}\right)^{-1}\left(D+L_{u}\right) D\left(2 D+\mathrm{ad}_{u}\right)^{-1},
$$

where $R_{u}$ and $L_{u}$ are the operators of right and left multiplications by $u$, respectively.

Note that in the commutative case (66) coincides with the modified KdV equation. It is easy to verify that (74) becomes the standard recursion operator of a modified $\mathrm{KdV}$ equation. All factors in (74) have to be regarded as operators acting on a (noncommutative) polynomial depending on $u, u_{x}, u_{x x}, \ldots$.

\section{CONCLUSION}

In this work we devoted ourselves in the construction of recursion operators when the Lax representation is given. We have shown that our approach can be easily generalized to all cases where the $L$ operator is a polynomial of $\lambda$. It would be interesting to generalize it for the cases of more complicated $\lambda$ dependence of $L$ as well as for the cases of $2+1$-dimensional equations, Toda-type lattices, and ordinary differential equations.

\section{ACKNOWLEDGMENTS}

We would like to thank Dr. Jing Ping Wang for reading the manuscript and pointing out some misprints. This work is partially supported by the Scientific and Technical Research Council of Turkey (TUBITAK). M. G. is a member of the Turkish Academy of Sciences (TUBA). V. S. is supported by Russian Foundation of Basic Researches (RFBR) Grant No. 99-01-00294 and INTAS. 


\section{APPENDIX A: EXAMPLE TO SEC. II A}

The Boussinesq equation,

$$
u_{t t}=-\frac{1}{3}\left(u_{4 x}+2\left(u^{2}\right)_{2 x}\right),
$$

can be expressed in the form of a pair of first-order evolution equations,

$$
\begin{gathered}
u_{t}=\mathrm{v}_{x}, \\
\mathrm{v}_{t}=-\frac{1}{3}\left(u_{3 x}+8 u u_{x}\right) .
\end{gathered}
$$

This system has a Lax pair,

$$
L=D^{3}+2 u D+u_{x}+\mathrm{v}, \quad A=\left(L^{2 / 3}\right)_{+} .
$$

To construct the recursion operator for this system, we use Eq. (12) with the differential operator,

$$
R_{n}=a_{n} D^{2}+b_{n} D+c_{n}
$$

By equating the coefficients of the powers of $D$ in (12), we find

$$
\begin{gathered}
a_{n}=\frac{2}{3} D^{-1}\left(u_{n}\right), \quad b_{n}=\frac{1}{3}\left(5 u_{n}+D^{-1}\left(\mathrm{v}_{n}\right)\right), \\
c_{n}=\frac{1}{9}\left(6 \mathrm{v}_{n}+8 u D^{-1}\left(u_{n}\right)+10 u_{n, x}\right),
\end{gathered}
$$

and after that we obtain the recursion operator of the form (40) for (A2) with

$$
\begin{gathered}
\mathcal{R}_{0}^{0}=3 \mathrm{v}+2 \mathrm{v}_{x} D^{-1}, \\
\mathcal{R}_{1}^{0}=D^{2}+2 u+u_{x} D^{-1}, \\
\mathcal{R}_{0}^{1}=-\left(\frac{1}{3} D^{4}+\frac{10}{3} u D^{2}+5 u_{x} D+3 u_{2 x}+\frac{16}{3} u^{2}+\left(\frac{2}{3} u_{3 x}+\frac{16}{3} u u_{x}\right) D^{-1}\right), \\
\mathcal{R}_{1}^{1}=3 \mathrm{v}+\mathrm{v}_{x} D^{-1} .
\end{gathered}
$$

\section{APPENDIX B: EXAMPLES TO SEC. II B}

\section{Sawada-Kotera equation}

The Lax pair for the Sawada-Kotera equation, ${ }^{28}$

$$
u_{t}=u_{5 x}+5 u u_{3 x}+5 u_{x} u_{2 x}+5 u^{2} u_{x}
$$

is given by

$$
L=D^{3}+u D, \quad A=\left(L^{5 / 3}\right)_{+} .
$$

In this example, $L^{\dagger}=-L$, where $L^{\dagger}=D^{-1} L^{*} D$ and $L$ is skew-symmetric, then we use (24). The operator $\widetilde{R}_{n}$ has the same form as (29), with the coefficients given by

$$
\begin{gathered}
a_{n}=\frac{1}{3} D^{-1}\left(u_{n}\right), \quad b_{n}=\frac{5}{3} u_{n}, \quad c_{n}=\frac{1}{9}\left(5 u D^{-1}\left(u_{n}\right)+29 u_{n, x}\right), \\
d_{n}=\frac{1}{9}\left(5 u_{x} D^{-1}\left(u_{n}\right)+14 u u_{n}+26 u_{n, 2 x}\right),
\end{gathered}
$$




$$
\begin{aligned}
e_{n}= & \frac{1}{27}\left(10 u_{2 x} D^{-1}\left(u_{n}\right)-2 D^{-1}\left(u_{2 x} u_{n}\right)-D^{-1}\left(u^{2} u_{n}\right)+5 u^{2} D^{-1}\left(u_{n}\right)\right. \\
& \left.+28 u_{n, 3 x}+32 u u_{n, x}+32 u_{x} u_{n}\right) \\
& f_{n}=0 .
\end{aligned}
$$

The recursion operator is given as

$$
\begin{aligned}
\mathcal{R}= & D^{6}+6 u D^{4}+9 u_{x} D^{3}+\left(9 u^{2}+11 u_{2 x}\right) D^{2}+\left(10 u_{3 x}+21 u u_{x}\right) D+5 u_{4 x}+16 u u_{2 x}+6 u_{x}^{2}+4 u^{3} \\
& +\left(u_{5 x}+5 u u_{3 x}+5 u_{x} u_{2 x}+5 u^{2} u_{x}\right) D^{-1}+u_{x} D^{-1}\left(u^{2}+2 u_{2 x}\right) .
\end{aligned}
$$

\section{DSI system}

The DSI system, ${ }^{25,26}$

$$
\begin{gathered}
u_{t}=3 \mathrm{vv}_{x}, \\
\mathrm{v}_{t}=2 \mathrm{v}_{3 x}+2 u \mathrm{v}_{x}+\mathrm{v} u_{x},
\end{gathered}
$$

has a Lax representation with

$$
\begin{gathered}
L=\left[D^{3}+(u+\mathrm{v}) D+\frac{1}{2}(u+\mathrm{v})_{x}\right]\left[D^{3}+(u-\mathrm{v}) D+\frac{1}{2}(u-\mathrm{v})_{x}\right], \\
A=\left(L^{1 / 2}\right)_{+} .
\end{gathered}
$$

Here $R_{n}$ is a differential operator of order 5 , and since $L$ is symmetric we again use Eq. (12). The expressions for the coefficients of the operator $R_{n}$ are very long and complicated. Hence we do not display them here. We find that the recursion operator $\mathcal{R}$ of this system is of the form (40), where

$$
\begin{aligned}
& \mathcal{R}_{0}^{0}=-4 D^{6}-24 u D^{4}-27 u_{x} D^{3}+2\left(-49 u_{2 x}-18 u^{2}+42 \mathrm{v}^{2}\right) D^{2}+10\left(-7 u_{3 x}-12 u u_{x}+30 \mathrm{vv}_{x}\right) D \\
&-26 u_{4 x}-82 u u_{2 x}-69 u_{x}^{2}+222 \mathrm{v}_{x}+141 \mathrm{v}_{x}^{2}-16 u^{3}+48 \mathrm{v}^{2} u \\
&+2\left(-2 u_{5 x}-10 u u_{3 x}-25 u_{x} u_{2 x}-10 u^{2} u_{x}+15 \mathrm{v}^{2} u_{x}+30 \mathrm{vv}_{3 x}+45 \mathrm{v}_{x} \mathrm{v}_{2 x}+30 u \mathrm{v}_{x}\right) D^{-1} \\
&+2 u_{x} D^{-1}\left(3 \mathrm{v}^{2}-2 u^{2}-u_{2 x}\right), \\
& \mathcal{R}_{1}^{0}=168 \mathrm{v} D^{4}+204 \mathrm{v} D^{3}+6\left(21 \mathrm{v}_{2 x}+32 u \mathrm{v}\right) D^{2}+6\left(40 \mathrm{v} u_{x}+7 \mathrm{v}_{3 x}+22 u \mathrm{v}_{x}\right) D \\
&+ 6\left(13 \mathrm{v} u_{2 x}+10 u_{x} \mathrm{v}_{x}+\mathrm{v}_{4 x}+5 u \mathrm{v}_{2 x}+4 \mathrm{v} u^{2}+12 \mathrm{v}^{3}\right)+108 \mathrm{v}_{x} D^{-1} \mathrm{v}+2 u_{x} D^{-1}\left(6 u \mathrm{v}+9 \mathrm{v}_{2 x}\right), \\
& \mathcal{R}_{0}^{1}= 56 \mathrm{v} D^{4}+268 \mathrm{v}_{x} D^{3}+2\left(243 \mathrm{v}_{2 x}+32 u \mathrm{v}\right) D^{2}+2\left(36 \mathrm{v} u_{x}+219 \mathrm{v}_{3 x}+106 u \mathrm{v}_{x}\right) D \\
&+2\left(27 \mathrm{v} u_{2 x}+92 u_{x} \mathrm{v}_{x}+99 \mathrm{v}_{a x}+99 u \mathrm{v}_{2 x}+4 \mathrm{v} u^{2}+12 \mathrm{v}^{3}\right)+2\left(10 \mathrm{v} u_{3 x}+35 u_{2 x} \mathrm{v}_{x}+45 u_{x} \mathrm{v}_{2 x}\right. \\
&\left.+10 u \mathrm{v} u_{x}+18 \mathrm{v}_{5 x}+30 u \mathrm{v}_{3 x}+10 u^{2} \mathrm{v}_{x}+15 \mathrm{v}^{2} \mathrm{v}_{x}\right) D^{-1}+2 \mathrm{v}_{x} D^{-1}\left(3 \mathrm{v}^{2}-2 u^{2}-u_{2 x}\right), \\
& \mathcal{R}_{1}^{1}= 108 D^{6}+216 u D^{4}+432 u_{x} D^{3}+6\left(81 u_{2 x}+18 u^{2}+22 \mathrm{v}^{2}\right) D^{2}+6\left(45 u_{3 x}+36 u u_{x}+70 \mathrm{vv}_{x}\right) D \\
&+3\left(18 u_{4 x}+18 u u_{2 x}+9 u_{x}^{2}+98 \mathrm{vv}_{2 x}+67 \mathrm{v}_{x}^{2}+32 u \mathrm{v}^{2}\right)+36\left(2 \mathrm{v}_{3 x}+2 \mathrm{v}_{x} u+\mathrm{v} u_{x}\right) D^{-1} \mathrm{v} \\
&+2 \mathrm{v}_{x} D^{-1}\left(6 u \mathrm{v}+9 \mathrm{v}_{2 x}\right) .
\end{aligned}
$$

\section{DSII system}

The DSII system, ${ }^{25,26}$ 


$$
\begin{gathered}
u_{t}=3 \mathrm{v}_{x}, \\
\mathrm{v}_{t}=-2\left(\mathrm{v}_{3 x}+u \mathrm{v}_{x}+\mathrm{v} u_{x}\right),
\end{gathered}
$$

has a Lax representation with

$$
\begin{gathered}
L=\left(D^{5}+u D^{3}+D^{3} u+\left(\mathrm{v}+\frac{1}{2} u^{2}\right) D+D\left(\mathrm{v}+\frac{1}{2} u^{2}\right)\right) D, \\
A=\left(L^{1 / 2}\right)_{+} .
\end{gathered}
$$

Since $L$ is symmetric we again use Eq. (12). In this case the operator $R_{n}$ is given as follows:

$$
R_{n}=a_{n} D^{5}+b_{n} D^{4}+c_{n} D^{3}+d_{n} D^{2}+e_{n} D,
$$

where

$$
\begin{gathered}
a_{n}=\frac{1}{3} D^{-1}\left(u_{n}\right), \quad b_{n}=\frac{5}{3} u_{n}, \\
c_{n}=\frac{1}{9}\left[5 u D^{-1}\left(u_{n}\right)+3 D^{-1}\left(\mathrm{v}_{n}\right)+29 u_{n, x}\right], \\
d_{n}=\frac{1}{9}\left[5 u_{x} D^{-1}\left(u_{n}\right)+26 u_{n, 2 x}+14 u u_{n}+12 \mathrm{v}_{n}\right], \\
e_{n}=\frac{1}{27}\left[5\left(2 u_{2 x}+u^{2}+3 \mathrm{v}\right) D^{-1}\left(u_{n}\right)-3 D^{-1}\left(\mathrm{v} u_{n}+u \mathrm{v}_{n}\right)+9 u D^{-1}\left(\mathrm{v}_{n}\right)\right. \\
\left.-2 D^{-1}\left(u_{2 x} u_{n}+\frac{1}{2} u^{2} u_{n}\right)+54 u_{x} u_{n}+28 u_{n, 3 x}+32\left(u u_{n, x}-u_{n} u_{x}\right)+42 \mathrm{v}_{n, x}\right] .
\end{gathered}
$$

The recursion operator (40) for the system can be found as ${ }^{29}$

$$
\begin{gathered}
\mathcal{R}_{0}^{0}=-D^{6}-6 u D^{4}-9 u_{x} D^{3}-\left(11 u_{2 x}+9 u^{2}+42 \mathrm{v}\right) D^{2}+\left(-10 u_{3 x}-21 u u_{x}-30 \mathrm{v}_{x}\right) D \\
-5 u_{4 x}-16 u u_{2 x}-6 u_{x}^{2}-60 \mathrm{v}_{2 x}-4 u^{3}-24 \mathrm{v} u+\left(-u_{5 x}-5 u u_{3 x}-5 u_{x} u_{2 x}\right. \\
\left.-5 u^{2} u_{x}-15 \mathrm{v} u_{x}-15 \mathrm{v}_{3 x}-15 u \mathrm{v}_{x}\right) D^{-1}-u_{x} D^{-1}\left(2 u_{2 x}+u^{2}+3 \mathrm{v}\right), \\
\mathcal{R}_{1}^{0}=-42 D^{4}-48 u D^{2}-87 u_{x} D-6\left(7 u_{2 x}+u^{2}-6 \mathrm{v}\right)+27 \mathrm{v}_{x} D^{-1}-3 u_{x} D^{-1} u, \\
\mathcal{R}_{0}^{1}=28 \mathrm{v} D^{4}+106 \mathrm{v}_{x} D^{3}+\left(165 \mathrm{v}_{2 x}+32 u \mathrm{v}\right) D^{2}+\left(54 \mathrm{v} u_{x}+132 \mathrm{v}_{3 x}+74 \mathrm{v}_{x} u\right) D+30 \mathrm{v} u_{2 x}+79 u_{x} \mathrm{v}_{x} \\
+54 \mathrm{v}_{4 x}+57 u \mathrm{v}_{2 x}+4 u^{2} \mathrm{v}-24 \mathrm{v}^{2}+\left(10 \mathrm{v} u_{3 x}+25 \mathrm{v}_{x} u_{2 x}+30 u_{x} \mathrm{v}_{2 x}+10 u \mathrm{v} u_{x}+9 \mathrm{v}_{5 x}+15 u \mathrm{v}_{3 x}\right. \\
\left.+5 u^{2} \mathrm{v}_{x}-15 \mathrm{v}_{x}\right) D^{-1}-\mathrm{v}_{x} D^{-1}\left(3 \mathrm{v}+u^{2}+2 u_{2 x}\right), \\
\mathcal{R}_{1}^{1}=27 D^{6}+54 u D^{4}+135 u_{x} D^{3}+3\left(54 u_{2 x}+9 u^{2}-22 \mathrm{v}\right) D^{2}+3\left(36 u_{3 x}+27 u u_{x}-28 \mathrm{v}_{x}\right) D \\
+3\left(9 u_{4 x}+9 u u_{2 x}+9 u_{x}^{2}-21 \mathrm{v}_{2 x}-16 \mathrm{v} u\right)-18\left(\mathrm{v}_{3 x}+u_{x} \mathrm{v}+\mathrm{v}_{x} u\right) D^{-1}-3 \mathrm{v}_{x} D^{-1} u .
\end{gathered}
$$

\section{DSIV system}

The DSIV system, ${ }^{25,26}$ which is also known as the Hirota-Satsuma system, ${ }^{30,31}$

$$
\begin{gathered}
u_{t}=\frac{1}{2} u_{3 x}+3 u u_{x}-6 \mathrm{vv}_{x}, \\
\mathrm{v}_{t}=-\mathrm{v}_{3 x}-3 u \mathrm{v}_{x},
\end{gathered}
$$

has Lax representation with

$$
L=\left(D^{2}+u+\mathrm{v}\right)\left(D^{2}+u-\mathrm{v}\right), \quad A=\left(L^{3 / 4}\right)_{+} .
$$


Since the operator $L$ is symmetric we use Eq. (12). In this case the operator $R_{n}$ has the same form as (39), with coefficients given by

$$
\begin{gathered}
a_{n}=\frac{1}{2} D^{-1}\left(u_{n}\right), \quad b_{n}=\frac{7}{4} u_{n}-\frac{1}{2} \mathrm{v}_{n}, \\
c_{n}=\frac{1}{8}\left[6 u D^{-1}\left(u_{n}\right)+2 D^{-1}\left(u u_{n}\right)-4 D^{-1}\left(\mathrm{vv}_{n}\right)+17 u_{n, x}-12 \mathrm{v}_{n, x}\right], \\
d_{n, x}=\frac{1}{16}\left[6 u_{2 x} D^{-1}\left(u_{n}\right)-12 \mathrm{v}_{2 x} D^{-1}\left(u_{n}\right)+30 u_{x} u_{n}-8 u_{x} \mathrm{v}_{n}+24 u u_{n, x}\right. \\
\left.+15 u_{n, 3 x}-12 \mathrm{v}_{x} \mathrm{v}_{n}-8 u \mathrm{v}_{n, x}-20 \mathrm{vv}_{n, x}-28 \mathrm{v}_{n, 3 x}\right] .
\end{gathered}
$$

The recursion operator (40) for the given system is

$$
\begin{gathered}
\mathcal{R}_{0}^{0}=\frac{1}{4} D^{4}+2 u D^{2}+3 u_{x} D+2 u_{2 x}+4\left(u^{2}-\mathrm{v}^{2}\right)+\left(3 u u_{x}-6 \mathrm{v}_{x}+\frac{1}{2} u_{3 x}\right) D^{-1}+u_{x} D^{-1} u, \\
\mathcal{R}_{1}^{0}=-5 \mathrm{v} D^{2}-4 \mathrm{v}_{x} D-\mathrm{v}_{2 x}-4 u \mathrm{v}-2 u_{x} D^{-1} \mathrm{v}, \\
\mathcal{R}_{0}^{1}=-\frac{5}{2} \mathrm{v}_{x} D-3 \mathrm{v}_{2 x}-\left(\mathrm{v}_{3 x}+3 u \mathrm{v}_{x}\right) D^{-1}+\mathrm{v}_{x} D^{-1} u, \\
\mathcal{R}_{1}^{1}=-D^{4}-4 u D^{2}-2 u_{x} D-4 \mathrm{v}^{2}-2 \mathrm{v}_{x} D^{-1} \mathrm{v} .
\end{gathered}
$$

\section{5. $N=3$ Hirota-Satsuma system}

This system is given by ${ }^{29}$

$$
\begin{gathered}
u_{t}=\frac{1}{4} u_{3 x}+3 u u_{x}+3\left(-\mathrm{v}^{2}+w\right)_{x}, \\
\mathrm{v}_{t}=-\frac{1}{2} \mathrm{v}_{3 x}-3 u \mathrm{v}_{x}, \\
w_{t}=-\frac{1}{2} w_{3 x}-3 u w_{x} .
\end{gathered}
$$

This is an example for the $N=3$ system that covers some other $N=2$ systems as special cases. For instance, letting $w=0$, we get DSIV and letting $\mathbf{v}=0$ we get DSIII systems.

The corresponding Lax pair is

$$
L=\left(D^{2}+2 u-2 \mathrm{v}\right)\left(D^{2}+2 u+2 \mathrm{v}\right)+4 w, \quad A=\left(L^{3 / 4}\right)_{+} .
$$

In this case the operator $L$ is symmetric and hence $R_{n}$ has the same form as (39), with the coefficients

$$
\begin{gathered}
a_{n}=D^{-1}\left(u_{n}\right), \quad b_{n}=\frac{7}{2} u_{n}+\mathrm{v}_{n}, \\
c_{n}=\frac{1}{4}\left[12 u D^{-1}\left(u_{n}\right)+4 D^{-1}\left(u u_{n}+w_{n}-2 \mathrm{vv}_{n}\right)+17 u_{n, x}+12 \mathrm{v}_{n, x}\right], \\
d_{n, x}=\frac{1}{8}\left[12 u_{2 x} D^{-1}\left(u_{n}\right)+24 \mathrm{v}_{2 x} D^{-1}\left(u_{n}\right)+60 u_{x} u_{n}+16 u_{x} \mathrm{v}_{n}+15 u_{n, 3 x}+48 u u_{n, x}+24 \mathrm{v}_{x} u_{n}\right. \\
\left.-40 \mathrm{v}_{x} \mathrm{v}_{n}+20 \mathrm{v}_{n, 3 x}+16 \mathbf{v v}_{n, x}+20 w_{n, x}\right] .
\end{gathered}
$$

The recursion operator is given by

$$
\mathcal{R}=\left(\begin{array}{ccc}
\mathcal{R}_{0}^{0} & \mathcal{R}_{1}^{0} & \mathcal{R}_{2}^{0} \\
\mathcal{R}_{0}^{1} & \mathcal{R}_{1}^{1} & \mathcal{R}_{2}^{1} \\
\mathcal{R}_{0}^{2} & \mathcal{R}_{1}^{2} & \mathcal{R}_{2}^{2}
\end{array}\right),
$$

where 


$$
\begin{gathered}
\mathcal{R}_{0}^{0}=\frac{1}{4} D^{4}+4 u D^{2}+6 u_{x} D+4\left(u_{2 x}+4 u^{2}-4 \mathrm{v}^{2}+4 w\right) \\
+4\left(\frac{1}{4} u_{3 x}+3 u u_{x}-6 \mathrm{v}_{x}+3 w_{x}\right) D^{-1}+4 u_{x} D^{-1} u, \\
\mathcal{R}_{1}^{0}=-2\left(5 \mathrm{v} D^{2}+4 \mathrm{v}_{x} D+\mathrm{v}_{2 x}+8 u \mathrm{v}+4 u_{x} D^{-1} \mathrm{v}\right), \\
\mathcal{R}_{2}^{0}=5 D^{2}+8 u+4 u_{x} D^{-1}, \\
\mathcal{R}_{0}^{1}=-5 u_{x} D-6 \mathrm{v}_{2 x}-2\left(\mathrm{v}_{3 x}+6 \mathrm{v}_{x} u\right) D^{-1}+4 \mathrm{v}_{x} D^{-1} u, \\
\mathcal{R}_{1}^{1}=-D^{4}-8 u D^{2}-4 u_{x} D+8\left(8 w-2 \mathrm{v}^{2}\right)-8 \mathrm{v}_{x} D^{-1} \mathrm{v}-8 D^{-1} w_{x}, \\
\mathcal{R}_{2}^{1}=4\left(\mathrm{v}_{x} D^{-1}+2 D^{-1} \mathrm{v}_{x}\right), \\
\mathcal{R}_{0}^{2}=-5 w_{x} D-6 w_{2 x}-2\left(\mathrm{v}_{3 x}+6 w_{x} u\right) D^{-1}+4 w_{x} D^{-1} u \\
\mathcal{R}_{1}^{2}=-16 \mathrm{v} D^{-1} w_{x}-8 w_{x} D^{-1} \mathrm{v}, \\
\mathcal{R}_{2}^{2}=-D^{4}-8 u D^{2}-4 u_{x} D+16\left(w-\mathrm{v}^{2}\right)+4 w_{x} D^{-1}+16 \mathrm{v} D^{-1} \mathrm{v}_{x} .
\end{gathered}
$$

\section{APPENDIX C: EXAMPLES TO SEC. III}

\section{Non-Abelian Schrödinger equation}

This is the system given by

$$
\begin{gathered}
u_{t}=-\frac{1}{2} u_{x x}+u \mathrm{v} u, \\
\mathrm{v}_{t}=\frac{1}{2} \mathrm{v}_{x x}+\mathrm{v} u \mathrm{v},
\end{gathered}
$$

where $u$ and $\mathrm{v}$ belong to $\mathcal{K}$ (see Example 6 for the notations). The Lax operator of (C1) is given by

$$
L=D+\left(\begin{array}{cc}
\mathbf{1} & 0 \\
0 & -\mathbf{1}
\end{array}\right) \lambda+\left(\begin{array}{cc}
0 & u \\
\mathbf{v} & 0
\end{array}\right)
$$

The corresponding formula (54) reduces to

$$
\left(\begin{array}{cc}
0 & u_{n+1} \\
\mathbf{v}_{n+1} & 0
\end{array}\right)=\lambda\left(\begin{array}{cc}
0 & u_{n} \\
\mathbf{v}_{n} & 0
\end{array}\right)+\left[R_{n}, L\right]
$$

where

$$
R_{n}=\left(\begin{array}{cc}
a_{n} & b_{n} \\
c_{n} & -a_{n}
\end{array}\right)
$$

The formula (C3) gives us both $a_{n}, b_{n}, c_{n}$ and the recursion operator $\mathcal{R}$. They are given by

$$
\begin{gathered}
a_{n}=\frac{1}{2} D^{-1}\left(u_{n} \mathrm{v}+u \mathrm{v}_{n}\right), \quad b_{n}=\frac{1}{2} u_{n}, \quad c_{n}=-\frac{1}{2} u_{n}, \\
\mathcal{R}=\frac{1}{2}\left(\begin{array}{cc}
-D+R_{u} D^{-1} R_{\mathrm{v}}+L_{u} D^{-1} L_{\mathrm{\vee}} & R_{u} D^{-1} L_{u}+L_{u} D^{-1} R_{u} \\
-L_{\mathrm{v}} D^{-1} R_{\mathrm{v}}-R_{\mathrm{v}} D^{-1} L_{\mathrm{v}} & D-R_{\mathrm{v}} D^{-1} R_{u}-L_{\mathrm{v}} D^{-1} L_{u}
\end{array}\right) .
\end{gathered}
$$




\section{Non-Abelian modified KdV equation}

The standard non-Abelian modified KdV equation is given by

$$
u_{t}=\frac{1}{4} u_{x x x}-\frac{3}{4} u_{x} u^{2}-\frac{3}{4} u^{2} u_{x} .
$$

The Lax representation of this equation is given

$$
L=D+\left(\begin{array}{cc}
0 & \mathbf{1} \\
\mathbf{1} & 0
\end{array}\right) \lambda+\left(\begin{array}{cc}
u & 0 \\
0 & -u
\end{array}\right)
$$

The recursion operator $\mathcal{R}$ can be found from (60) and (61). In our case the automorphism $\sigma$ is the same as in Example 6, and formulas (60) and (61) give us

$$
\left(\begin{array}{cc}
0 & u_{n+1} \\
\mathbf{v}_{n+1} & 0
\end{array}\right)=\lambda^{2}\left(\begin{array}{cc}
0 & u_{n} \\
\mathbf{v}_{n} & 0
\end{array}\right)+\left[R_{n}, L\right]
$$

where

$$
R_{n}=\left(\begin{array}{cc}
0 & a_{n} \\
b_{n} & 0
\end{array}\right) \lambda+\left(\begin{array}{cc}
c_{n} & 0 \\
0 & d_{n}
\end{array}\right)
$$

Using (C9) we find $a_{n}, b_{n}, c_{n}, d_{n}$ from the following:

$$
\begin{gathered}
b_{n}-a_{n}=u_{n}, \quad-a_{n, x}-a_{n} u-u a_{n}+c_{n}-d_{n}=0, \\
-b_{n, x}+b_{n} u+u b_{n}+d_{n}-c_{n}=0, \quad d_{n, x}+c_{n, x}=\left[c_{n}-d_{n}, u\right], \\
u_{n+1}=d_{n, x}+\left[d_{n}, u\right] .
\end{gathered}
$$

The resulting recursion operator is given by

$$
\mathcal{R}=\frac{1}{4}\left(D-\operatorname{ad}_{u} \cdot D^{-1} \cdot \operatorname{ad}_{u}\right)\left(D-\left(L_{u}+R_{u}\right) D^{-1}\left(L_{u}+R_{u}\right)\right) .
$$

${ }^{1}$ P. J. Oliver, Applications of Lie Groups to Differential Equations, Graduate Texts in Mathematics, 2nd ed. (SpringerVerlag, New York, 1993), Vol. 107.

${ }^{2}$ P. J. Olver, J. Math. Phys. 18, 1212 (1977).

${ }^{3}$ M. Gürses and A. Karasu, J. Math. Phys. 36, 3485 (1995).

${ }^{4}$ M. Gürses and A. Karasu, Phys. Lett. A 214, 21 (1996).

${ }^{5}$ M. Gürses and A. Karasu, J. Math. Phys. 39, 2103 (1998).

${ }^{6}$ M. Gürses and A. Karasu, Phys. Lett. A 251, 247 (1999).

${ }^{7}$ S. I. Svinolupov, Theor. Math. Phys. 87, 391 (1991).

${ }^{8}$ J. Krasil'shchik, Contemp. Math. 219, 121 (1998).

${ }^{9}$ A. S. Fokas and R. L. Anderson, J. Math. Phys. 23, 1066 (1982).

${ }^{10}$ A. S. Fokas, Stud. Appl. Math. 77, 253 (1987).

${ }^{11}$ A. P. Fordy and J. Gibbons, J. Math. Phys. 22, 1170 (1980).

${ }^{12}$ P. M. Santini and A. S. Fokas, Commun. Math. Phys. 115, 375 (1988).

${ }^{13}$ A. S. Fokas and P. M. Santini, Commun. Math. Phys. 116, 449 (1988).

${ }^{14}$ W. Symes, J. Math. Phys. 20, 721 (1979).

${ }^{15}$ M. Adler, Invent. Math. 50, 219 (1979).

${ }^{16}$ I. Ya. Dorfman and A. S. Fokas, J. Math. Phys. 33, 2504 (1992).

${ }^{17}$ A. S. Fokas and I. M. Gel'fand, in Important Developments in Soliton Theory, Springer Series in Nonlinear Dynamics, edited by A. S. Fokas and V. E. Zakharov (Springer-Verlag, Berlin, 1993), pp. 259-282.

${ }^{18}$ M. Antonowicz and A. P. Fordy, in Nonlinear Evolution Equations and Dynamical Systems (NEEDS'87), edited by J. Leon (World Scientific, Singapore, 1988), pp. 145-160.

${ }^{19}$ M. Antonowicz and A. P. Fordy, in Soliton Theory: A Survey of Results, edited by A. P. Fordy (Manchester University Press, Manchester, England, 1990). See also the related references therein.

${ }^{20}$ I. M. Gel'fand and L. A. Dikii, Funct. Anal. Appl. 10, 13 (1976).

${ }^{21}$ F. Magri, J. Math. Phys. 19, 1156 (1978).

${ }^{22}$ V. V. Sokolov, Sov. Math. Dokl. 30, 44 (1984). 
${ }^{23}$ Here we note that in 1980 that Shabat and Sokolov independently found the recursion operator for the Sawada-Kotera equation. This result was published in Ref. 24. In Ref. 22, Sokolov found the recursion operator for the KricheverNovikov equation.

${ }^{24}$ N. H. Ibragimov, Transformation Groups Applied to Mathematical Physics (Reidel, Boston, 1985).

${ }^{25}$ V. G. Drinfeld and V. V. Sokolov, J. Sov. Math. 30, 1975 (1985).

${ }^{26}$ V. G. Drinfeld and V. V. Sokolov, Proc. Sobolev Sem. Novosibirsk 2, 5 (1981) (in Russian).

${ }^{27}$ I. Z. Golubchik and V. V. Sokolov, Theor. Math. Phys. 112, 1097 (1997).

${ }^{28}$ K. Sawada and T. Kotera, Prog. Theor. Phys. 51, 1355 (1974).

${ }^{29}$ J. Springael, X. B. Hu, and I. Loris, J. Phys. Soc. Jpn. 65, 1222 (1996).

${ }^{30}$ J. Satsuma and R. Hirota, J. Phys. Soc. Jpn. 51, 3390 (1982).

${ }^{31}$ G. Wilson, Phys. Lett. A 89, 332 (1982). 